\title{
Artigo/Article
}

\section{Fatores de risco associados a alterações renais em pacientes infectados por HIV-1}

\author{
Risk factors related to renal disorders in HIV-1 infected patients
}

\section{Lauro Ferreira Silva Pinto Neto ${ }^{1,2}$,Andressa Costa $\mathrm{Braga}^{3}$, Juliana Alvarenga Rocha ${ }^{3}$, Nilo Fernando Rezende Vieira ${ }^{1}$ e Angélica Espinosa Miranda ${ }^{2}$ \\ RESUMO \\ Introdução: A doença renal é uma das principais comorbidades envolvendo pacientes infectados com o HIV, em razão da melhora da sobrevida proporcionada pela terapêutica antirretroviral. $\mathrm{O}$ \\ INTRODUÇÃO} objetivo deste estudo foi detectar fatores de risco, possivelmente correlacionados com função renal alterada, em pacientes infectados pelo HIV. Métodos: Estudo transversal foi realizado em 254 pacientes infectados pelo HIV, atendidos em ambulatório na Santa Casa de Vitória. Eles foram entrevistados e submetidos a coletas de amostras de sangue para contagem de células CD4, quantificação de carga viral do HIV-1, dosagens de glicose, lipídeos e creatinina. A proteinúria foi avaliada em amostra de primeira urina da manhã. A filtração glomerular foi estimada com as fórmulas de modified diet in renal disease (MDRD) simplificada e CockcroftGault. Resultados: Cento e três (40,6\%) pacientes tinham alguma anormalidade no exame de urina, sendo proteinúria o achado mais comum $(46 ; 18,1 \%$ pacientes). Vinte e cinco $(9,8 \%)$ pacientes tinham filtração glomerular estimada inferior a $60 \mathrm{ml} / \mathrm{min} / 1.73 \mathrm{~m}^{2}$ de acordo com MDRD. A análise de regressão logística multivariada mostrou que baixa filtração glomerular foi positivamente correlacionada com raça negra [OR 9,6 (IC95\% 1,28-23,80)], hipertensão arterial sistêmica [OR 3,3 (IC95\% 1,28-23,81)], idade acima de 51 anos [OR 3,3 (IC95\%1,11-9,90)], proteinúria [OR 5,2 \{IC95\% 1,67-16,25\}]; hematúria [OR 3,2 (1,12-9,29)] e negativamente com pacientes em uso de zidovudina [OR 0,2 (0,04-0,78)]. Conclusões: Os fatores de risco tradicionais para doença renal como raça negra, hipertensão arterial e idade avançada foram correlacionados com menor filtração glomerular estimada em nossos pacientes.

Palavras-chaves: HIV. Doença renal crônica. Modified diet in renal disease.

\begin{abstract}
Introduction: Renal disease has emerged as one of the primary comorbid conditions affecting HIV-infected patients, mainly because antiretroviral therapy has improved survival. This study aimed to detect risk factors possibly associated with altered renal function in HIV-infected patients. Methods: A cross-sectional study was conducted involving $254 \mathrm{HIV}$-infected patients attending an outpatient clinic at Santa Casa de Vitoria < They were interviewed and blood samples were collected for CD4 cell counts, HIV-1 viral load, glucose, lipids and creatinine measurements. Urine protein was evaluated in the first voiding urine sample. Glomerular filtration was estimated by simplified modified diet in renal disease (MDRD) and CockcroftGault formulas. Results: One hundred and three (40.6\%) patients presented some urinary abnormality, and proteinuria was the most common finding (46; $18.1 \%$ patients). Twenty-five (9.8\%) patients showed estimated glomerular filtration below $60 \mathrm{ml} / \mathrm{min} / 1.73 \mathrm{~m}^{2}$ by MDRD. Multivariate logistic regression showed that low glomerular filtration was positively correlated with black race [OR 9.6 (IC95\% 1.28-23.80)], arterial hypertension [OR 3.3 (IC95\% 1.2823.81)], age over fifty-one years-old [OR 3.3 (IC95\%1.11-9.90)], proteinuria [OR 5.2 \{IC95\% 1.67-16.25\}]; hematuria [OR $3.2(1.12-9.29)$ ] and negatively correlated with patients using zidovudine [OR $0.2(0.04-0.78)]$. Conclusions: Traditional risk factors for renal disease, such as black race, arterial hypertension and advancing age were correlated with low estimated glomerular filtration in the present patient sample.
\end{abstract}

Keywords: HIV. Chronic renal disease. Modified diet in renal disease.

1. Departamento de Clínica Médica, Escola Superior de Ciências, Santa Casa de Misericórdia de Vitória, Vitória, ES. 2. Programa de Pós-Graduação em Doenças Infecciosas, Universidade Federal do Espírito Santo, Vitória, ES. 3. Curso de Medicina, Escola Superior de Ciências, Santa Casa de Misericórdia de Vitória, Vitória, ES.

Endereço para correspondência: Dr. Lauro Ferreira da Silva Pinto Neto. R. Chapot Presvot 100/1701, Praia do Canto, 29055-410 Vitória, ES.

Tel: 5527 3345-2123

e-mail: lauro.neto@emescam.br

Recebido para publicação em 13/04/2010

Aceito em 18/10/2010
Próximo a se completarem 30 anos da existência da pandemia causada pelo HIV-1, a extraordinária sobrevida obtida através da terapia antirretroviral, com redução da incidência e mortalidade por infecções oportunistas, tem desviado o foco da atenção para comorbidades outras que afetam a população infectada e também podem causar mortes $^{1}$. O envelhecimento das pessoas soropositivas para HIV-1 e o aumento de novas infecções em pessoas acima dos 50 anos $^{2}$ também compelem os profissionais envolvidos no cuidado a estes pacientes a atentarem para as doenças crônicas comuns nessas faixas etárias, entre as quais as doenças renais.

No Brasil, o Departamento de DST, AIDS e Hepatites do Ministério da Saúde, nas diretrizes em vigor, recomenda realização, ao menos anualmente, de exames simples de urina e de avaliações de função renal nos pacientes infectados ${ }^{3}$. A Sociedade Americana de Doenças Infecciosas (IDSA) recomenda que todos os pacientes, no momento do diagnóstico da infecção pelo HIV, sejam submetidos a rastreamento de proteinúria em amostra de urina e ao cálculo estimado da função renal, por meio das fórmulas disponíveis, como as de Cockcroft-Gault, ou a da dieta modificada em doença renal, do inglês modified diet in renal disease (MDRD) simplificada ${ }^{4}$.

A nefropatia associada ao HIV foi, originalmente descrita, em 1984, como uma glomeruloesclerose focal e segmentar, com apresentação clínica de proteinúria nefrótica e rápido declínio da função renal ${ }^{5,6}$. Sua incidência aumentou em meados da década de $1990^{7}$, e tem permanecido estável ao longo dos últimos anos, após um declínio inicial atribuído ao sucesso da moderna terapia antiviral ${ }^{8}$. No entanto, em um contexto mais amplo, a prevalência de doença renal parece estar aumentando na população infectada pelo HIV-1, segundo alguns autores ${ }^{9,10}$. Estima-se que $17 \%$ dos infectados tenham doença renal crônica, que parece estar associada ao fato dos pacientes serem mais idosos, à infecção avançada pelo HIV-1, aos níveis mais elevados de carga viral e aos níveis mais baixos de linfócitos TCD4, a doenças 
vasculares e aos distúrbios metabólicos, à raça negra ${ }^{11} \mathrm{e}$, ainda, ao uso de alguns antivirais, como indinavir e tenofovir ${ }^{12}$. Diabetes melito e hipertensão arterial sistêmica são os principais fatores de risco para doença renal na população em geral, aumentando em 10 vezes o risco de doença renal crônica ${ }^{11}$ e são cada vez mais frequentes nos soropositivos HIV.

Um estudo de coorte mostrou a prevalência de diabetes em 14\% na população HIV positivo ${ }^{13}$ e estima-se que a prevalência de hipertensão arterial seja de $12 \%$ a $21 \%$, na mesma população ${ }^{14}$. A coinfecção com o vírus da hepatite $\mathrm{C}$ está associada com risco de doença renal demonstrada em alguns estudos observacionais ${ }^{11}$, embora possam existir fatores de risco concorrentes nas duas infecções. Verificou-se que alguma anormalidade da função renal pode ser detectada em até 30\% dos pacientes com $\mathrm{HIV}-1^{4}$. Sem intervenção precoce, um percentual significativo destes pacientes irá sofrer progressão da doença renal ${ }^{15}$.

Estudo patrocinado pelo Centers for Disease Control (CDC) mostrou que a presença de proteinúria e/ou a elevação dos níveis de creatinina eram positivamente associadas com maior risco de morte (risco ajustado de 2,5) em mulheres infectadas ${ }^{16}$. Do mesmo modo, anormalidades laboratoriais de exames renais estão associadas a taxas de hospitalização maiores. Essas alterações estão vinculadas a maior mortalidade, seja antes ou depois da terapia antirretroviral ${ }^{4}$.

Ainda são escassos os relatos em nosso meio de alterações renais em pacientes infectados pelo HIV- ${ }^{17}$, a despeito da importância e abrangência do Programa Brasileiro de HIV/AIDS.

O objetivo deste trabalho foi analisar a correlação dos fatores de risco para perda da função renal em uma população infectada pelo HIV em seguimento em um serviço especializado em nosso meio.

\section{MÉTODOS}

O Programa de HIV/AIDS da Santa Casa de Vitória é o segundo maior serviço especializado do estado, com mais de 600 pacientes cadastrados em uso de antirretrovirais. Entre junho e novembro de 2009, todos os pacientes que realizavam coleta regular de CD4/ CD8 e carga viral de HIV-1 foram abordados e, após assinatura do termo de consentimento informado, foram incluídos em um estudo transversal e submetidos à pesagem, aferição de pressão arterial e interrogatório de uso de anti-hipertensivos, antidiabéticos e ou drogas nefrotóxicas. Os pacientes também foram submetidos aos exames de glicemia, lipídeos totais, creatinina sanguinea, creatinina urinária, e exame simples de urina para detecção de proteinúria.

O desenho inicial do estudo previa a determinação da razão proteína/creatinina na amostra de urina matinal, mas, por motivos de ordem técnica, só foi possível a determinação da proteína no exame simples de urina. Os pacientes com intercorrências clínicas, caracterizadas por febre ou infecção que interferissem nos resultados de contagem de linfócitos T CD4/CD8 foram excluídos do estudo.

A filtração glomerular foi estimada através das fórmulas de Cockcroft-Gault e MDRD simplificada. A primeira fórmula tem sido usada desde 1976 e é a mais cotada na adequação de doses de antirretrovirais, de acordo com a maioria das diretrizes vigentes. A equação MDRD foi elaborada em 1999 e, posteriormente, simplificada em uma versão que tem sido recomendada como a mais fiel preditora da função renal, especialmente nos casos em que a mesma está reduzida em relação aos níveis normais ${ }^{18}$. Ela foi usada como variável preditiva da função renal.
As variáveis foram submetidas à avaliação estatística com o uso do programa SPSS, versão 17 para Windows. Foi realizada uma análise descritiva, com cálculo de frequência para variáveis qualitativas e cálculo de média e desvio padrão para variáveis quantitativas. As possíveis associações entre déficit de filtração glomerular estimada pela fórmula MDRD com as variáveis demográficas, comportamentais e clínicas foram testadas por meio de testes de qui-quadrado com correção de Yates ou de Fischer quando apropriado. Odds ratio e intervalos de confiança foram calculados em análises bivariadas para estimar o grau de associação entre o déficit da função renal e os potenciais fatores de risco. Foi aplicada análise multivariada de regressão logística para estimar o efeito de uma variável, ao mesmo tempo em que se controla o efeito das demais, na probabilidade de apresentar queda da filtração glomerular estimada pelo MDRD, fixando-se o corte em $60 \mathrm{ml} / \mathrm{min}$.

\section{Considerações éticas}

Este projeto foi submetido ao Comitê de Ética da Escola de Ciências de Saúde da Santa Casa de Vitoria e todos os pacientes foram convidados a participar em caráter voluntario. Todos os casos com alterações mais significativas foram encaminhados ao serviço especializado e receberam tratamento.

\section{RESULTADOS}

De 271 pacientes que compareceram para a coleta regular de exames de linfócitos TCD4/TCD8 e PCR quantitativo de HIV-1 e que eram elegíveis para o estudo, 11 se recusaram a assinar o termo de consentimento informado e seis não tiveram todos os dados disponibilizados para o estudo. Entre os 254 (93,7\%) pacientes incluídos no estudo, 135 (53,1\%) eram do sexo masculino, 117 (46,1\%) brancos, $56(31,9 \%)$ negros e $81(22 \%)$ pardos, com idade média de 42,76 (DP 10,29) anos. Cinquenta e oito $(22,8 \%)$ pacientes tinham mais de 51 anos. O tempo médio de diagnóstico da infecção pelo HIV era de 7,13 (DP 4,33) anos.

A média estimada de filtração glomerular foi de 97 (DP 27,49) $\mathrm{ml} / \mathrm{min} / 1.73 \mathrm{~m}^{2}$ através da formula de Crockoft-Gault e de 90,95 (DP $26,72) \mathrm{ml} / \mathrm{min} / 1.73 \mathrm{~m}^{2}$ na MDRD simplificada. Vinte e cinco (9,8\%) pacientes tinham filtração glomerular abaixo de $60 \mathrm{ml} / \mathrm{min} / 1.73 \mathrm{~m}^{2}$ pela MDRD simplificada enquanto 17 (6,7\%) apresentavam este parâmetro de filtração glomerular através da formula de CrockoftGault.

A Tabela 1 descreve a correlação da função renal com variáveis demográficas e clínicas. A coinfecção pelo vírus da hepatite $\mathrm{C}$ foi observada em apenas $13(5,1 \%)$ pacientes.

Os pacientes apresentavam contagem média de linfócitos TCD4 de 544,51 (DP 311) células/ml, e 64,2\% estavam com PCR quantitativo do HIV-1 abaixo de 50 cópias/ml pelo método bDNA. Cinquenta e três $(20,9 \%)$ pacientes eram hipertensos e 48 (18,8\%) pacientes apresentavam níveis glicêmicos maiores que $99 \mathrm{mg} / \mathrm{dL}$, dos quais $13(5,1 \%)$ acima de $126 \mathrm{mg} / \mathrm{dL}$.

Um total de $214(84,3 \%)$ pacientes estava em uso de terapêutica antirretroviral, dos quais, $126 \mathrm{em}$ uso de IP/r, sendo o lopinavir/r (87 pacientes) o mais frequente, seguido do atazanavir/r (32 pacientes). Cem pacientes estavam em uso de não nucleosídeos, sendo efavirenz o mais frequente (71 pacientes), seguido da nevirapina em 29 pacientes. A Tabela 2 mostra que o análogo de nucelosídeo mais usado era a lamivudina (205 pacientes) seguido pela zidovudina (122 pacientes) e pelo tenofovir (92 pacientes). 
TABELA 1 - Frequência dos fatores de risco e associações com a queda da função renal nos 254 pacientes atendidos na Santa Casa de Misericórdia de Vitória, Estado de Espírito Santo.

\begin{tabular}{|c|c|c|c|c|c|c|}
\hline \multirow[b]{2}{*}{ Variáveis } & \multicolumn{2}{|c|}{ Total } & \multicolumn{2}{|c|}{$\mathrm{MDRD}<60$} & \multirow[b]{2}{*}{ OR (IC95\%) } & \multirow[b]{2}{*}{ Valor $\mathrm{p}$} \\
\hline & $\mathrm{n}^{\mathrm{o}}$ & $\%$ & $\mathrm{n}^{\mathrm{o}}$ & $\%$ & & \\
\hline \multicolumn{7}{|l|}{ Idade (anos) } \\
\hline$\leq 50$ & 196 & 77,2 & 14 & 7,1 & 1 & 0,008 \\
\hline$\geq 51$ & 58 & 22,8 & 11 & 19,0 & $3,0(1,30-7,14)$ & \\
\hline \multicolumn{7}{|l|}{ Sexo } \\
\hline masculino & 135 & 53,1 & 6 & 4,4 & 1 & 0,002 \\
\hline feminino & 119 & 6,9 & 19 & 16,0 & $4,1(1,57-10,64)$ & \\
\hline \multicolumn{7}{|l|}{ Cor da pele } \\
\hline negra & 56 & 22,0 & 1 & 1,8 & $7,6(1,10-58,82)$ & 0,021 \\
\hline outras & 198 & 78,0 & 24 & 12,1 & 1 & \\
\hline \multicolumn{7}{|c|}{ Hipertensão arterial } \\
\hline $\operatorname{sim}$ & 53 & 20,9 & 10 & 18,9 & $2,9(1,21-6,85)$ & 0,013 \\
\hline não & 201 & 79,1 & 15 & 7,5 & 1 & \\
\hline \multicolumn{7}{|l|}{ Glicemia } \\
\hline$\leq 125$ & 241 & 94,9 & 24 & 10,0 & 1 & 0,627 \\
\hline$\geq 126$ & 13 & 5,1 & 1 & 7,7 & $1,3(0,17-10,66)$ & \\
\hline \multicolumn{7}{|l|}{ Proteinúria } \\
\hline presente & 46 & 18,1 & 11 & 23,9 & $4,4(1,83-10,37)$ & 0,001 \\
\hline ausente & 208 & 81,9 & 14 & 6,7 & 1 & \\
\hline \multicolumn{7}{|l|}{ Hematúria } \\
\hline presente & 59 & 23,2 & 11 & 18,6 & $2,9(1,27-6,94)$ & 0,010 \\
\hline ausente & 195 & 76,8 & 14 & 7,2 & 1 & \\
\hline \multicolumn{7}{|l|}{ Cristais no EAS } \\
\hline presente & 34 & 13,4 & 7 & 20,6 & $2,9(1,11-7,61)$ & 0,024 \\
\hline ausente & 220 & 86,6 & 18 & 8,2 & 1 & \\
\hline \multicolumn{7}{|l|}{ Teste para VHC } \\
\hline reativo & 13 & 5,1 & 1 & 7,7 & $1,2(0,14-9,59)$ & 0,603 \\
\hline não reativo & 241 & 94,9 & 16 & 6,6 & 1 & \\
\hline \multicolumn{7}{|l|}{ Carga viral } \\
\hline indetectável & 163 & 64,2 & 20 & 12,3 & 1 & 0,082 \\
\hline detectável & 91 & 35,8 & 5 & 5,5 & $2,4(0,87-6,64)$ & \\
\hline \multicolumn{7}{|l|}{ CD4 } \\
\hline$<200 \mathrm{cel} / \mathrm{ml}$ & 28 & 11,0 & 5 & 17,9 & $2,2(0,77-6,53)$ & 0,131 \\
\hline$\geq 200 \mathrm{cel} / \mathrm{ml}$ & 226 & 89,0 & 20 & 8,8 & 1 & \\
\hline
\end{tabular}

MDRD: modified diet in renal disease, EAS: elementos anormais sedimentados, VHC: vírus da hepatite $\mathrm{C}, \mathrm{OR}$ : odds ratio.

TABELA 2 - Frequência de uso de antirretrovirais e associações com a queda da função renal nos 254 pacientes atendidos na Santa Casa de Misericórdia de Vitória, Estado de Espírito Santo.

\begin{tabular}{lcccccccc}
\hline & \multicolumn{2}{c}{ Total } & & \multicolumn{2}{c}{ MDRD $<60$} & & \\
\cline { 2 - 3 } Variáveis & $\mathrm{n}^{\circ}$ & $\%$ & & $\mathrm{n}^{\circ}$ & $\%$ & & OR (IC95\%) & Valor $\mathrm{p}$ \\
\hline Zidovudina & 122 & 48,0 & & 6 & 4,9 & $0,3(0,12-0,80)$ & 0,011 \\
Lamivudina & 205 & 80,7 & & 20 & 9,8 & $0,9(0,34-2,68)$ & 0,925 \\
Efavirenz & 71 & 28,0 & 2 & 2,8 & $0,2(0,05-0,88)$ & 0,019 \\
Tenofovir & 92 & 36,2 & & 13 & 14,1 & $2,1(0,89-4,72)$ & 0,084 \\
Lopinavir & 87 & 34,3 & & 15 & 17,2 & $3,3(1,40-7,63)$ & 0,004 \\
Nevirapina & 29 & 11,4 & & 1 & 3,4 & $0,3(0,04-2,30)$ & 0,219 \\
Atazanavir & 32 & 12,6 & & 4 & 12,5 & $1,4(0,44-4,28)$ & 0,533 \\
Indinavir & 1 & 0,4 & & 1 & 100,0 & & 0,098 \\
Abacavir & 3 & 1,2 & & 1 & 33,3 & $4,7(0,41-54,01)$ & 0,268
\end{tabular}

${ }^{*}$ Cálculo não realizado, pois uma das células estava vazia, MORD: modified diet in renal disease, OR: odds ratio.
Cento e três $(40,6 \%)$ pacientes tinham alguma alteração no exame simples de urina caracterizado por proteinúria (46 pacientes; $18,1 \%$ ) ou hemoglobinúria/hematúria, ou mesmo cristais (o restante). A proteinúria foi mais frequente nos pacientes com filtração glomerular estimada menor que $60 \mathrm{ml} / \mathrm{min} / 1,73 \mathrm{~m}^{2}$ pela $\operatorname{MDRD}(44 \% \times 4,3 \%, \mathrm{p}=0,001)$ nos pacientes em uso de lamivudina $(21 \%$ x $6,1 \% \mathrm{p}=0,013)$ e lopinavir/r $(29,9 \%$ x $12 \% \mathrm{p}=0,001)$. A proteinúria mostrou tendência de maior frequência nos pacientes com doença mais avançada (CD4 menor que 200, 32,1\% x 16,4\% $\mathrm{p}=0,064)$ e foi um pouco mais frequente nos pacientes em uso de tenofovir $(22,8 \%$ x 15,4\%) embora sem significância estatística $(p=0,175)$.

A análise multivariada de regressão logística mostrou que a filtração glomerular menor que $60 \mathrm{ml} /$ minuto na fórmula MDRD simplificada teve correlação positiva com a raça negra [OR 9,6 (IC95\% 1,28-23,80)], pressão arterial diastólica acima de 90mmHg [OR 3,3 (IC95\% 1,28-23,81)]; idade acima de 51 anos [OR 3,3 (IC95\%1,11-9,90)]; proteinúria [OR 5,2 \{IC95\% 1,67-16,25\}]; hematúria $[$ OR $3,2(1,12-9,29)]$ e correlação negativa com o uso de zidovudine [OR 0,2 (0,04-0,78)] (Tabela 3).

TABELA 3 - Fatores independentemente associados com a queda da função renal (fórmula MDRD) no modelo final de regressão logística.

\begin{tabular}{lcc}
\hline Variáveis & OR (IC95\%) & Valor p \\
\hline Cor da pele (negra $v$ s outras) & $9,6(1,28-23,80)$ & 0,047 \\
Idade $(\geq 51$ vs $\leq 50$ anos) & $3,3(1,11-9,90)$ & 0,032 \\
Proteinúria & $5,2(1,67-16,25)$ & 0,004 \\
Hematúria & $3,2(1,12-9,29)$ & 0,030 \\
Hipertensão arterial & $5,5(1,28-23,81)$ & 0,022 \\
Uso zidovudina & $0,2(0,04-0,78)$ & 0,011 \\
\hline
\end{tabular}

Variáveis que entraram no modelo: glicemia, pressão_arterial, cor_pele, idade_ $\geq 51$, CD4, lopinavir.EFV: efavirenz, AZT: zidovudine, proteína, hemácias, cristais. MDRD: modified diet in renal disease, OR: odds ratio, IC 95\%: intervalo de confiança 95\%.

\section{DISCUSSÃO}

Nosso estudo reforçou a importância da implementação cuidadosa do rastreamento da função renal na prática clínica de um serviço especializado no atendimento a HIV/AIDS em nosso meio. Mereceu destaque o elevado percentual de pacientes (40,6\%) com alguma alteração no exame simples de urina, em especial proteinúria, o que vem sendo relatado na literatura ${ }^{11}$. Mais recentemente, alguns autores têm realçado uma diretriz do National Kidney Foundation que recomenda rastreamento de proteinúria através da razão albumina/ creatinina, de preferência na primeira urina da manha ${ }^{19,20}$. No entanto, isto envolve técnica um pouco mais sofisticada que uso de uma simples fita de urina, e não foi possível em nosso estudo, limitando a precisão de nosso achado. De qualquer modo, o simples relato de proteinúria no exame simples de urina é um dos mais precoces indicadores de vários tipos de doença renal, relatado em até um terço dos pacientes infectados pelo HIV-1 e associado à progressão mais rápida para AIDS e morte ${ }^{19}$.

O padrão ouro para determinação da proteinúria e mesmo da filtração glomerular, a coleta de urina de $24 \mathrm{~h}$, é considerado um método de difícil aplicabilidade no conjunto de pacientes na prática clínica, razão pela qual, diversos autores têm recomendado a utilização de fórmulas para estimar a função renal. Privilegiamos o uso da MDRD simplificada, por esta ter se mostrado com maior precisão em pacientes com algum déficit de função renal ${ }^{18}$. A importância de rastrear a alteração precoce da função renal se destaca 
em razão dos estudos que têm mostrado o impacto da doença renal crônica em pacientes infectados pelo HIV-1, quando se relata que pacientes com filtração glomerular menor que $15 \mathrm{ml} / \mathrm{min} / 1.73 \mathrm{~m}^{2}$ tem até 6 vezes maior risco de morte ${ }^{19}$, comparados com pacientes com filtração glomerular maior que $60 \mathrm{ml} / \mathrm{min} / 1.73 \mathrm{~m}^{2}$. É possível que esta diferença importante se explique em parte pela dificuldade de adequação correta de doses de antirretrovirais em pacientes com queda de função renal, com consequente utilização de níveis mais baixos das drogas nos mesmos ${ }^{19}$. Apenas uma minoria dos pacientes HIV-1 positivos com algum déficit de função renal são identificados, de acordo com estudo recente ${ }^{21}$, o que só reforça a imensa necessidade de fazê-lo.

Em acordo com o já descrito na literatura, são os fatores de risco tradicionais para doença renal crônica que se mostraram associados de modo independente com a queda da função renal nos pacientes infectados pelo HIV-1 em nosso estudo. A raça negra (OR: 9,6), a hipertensão arterial sistêmica (OR: 5,5) e a idade maior que 50 anos (OR: 3,2), além da proteinúria (OR: 5,2) e hematúria (OR: 3,2 ) evidenciaram significância estatística no modelo de regressão usado. O envelhecimento da população infectada pelo HIV-1, com a melhoria da sobrevida com a terapêutica antirretroviral, além do aumento da prevalência na população mais idosa, só reforçam a necessidade dos cuidadores de HIV/AIDS estarem mais alertas à função renal de seus pacientes.

Interessante o achado que tivemos do aparente efeito protetor do uso de zidovudina na preservação da função renal, considerando-se que o grupo em uso da mesma mostrou associação negativa com queda da função renal, na análise de regressão logística (OR de 0,2, Tabela 3). O estudo Aproco-Pilote, de uma coorte européia de pacientes infectados ${ }^{22}$, recentemente, publicado, além de mostrar uma forte associação com idade e função renal, demonstrou uma melhora da função renal após inicio da terapêutica e sugere que pequenas alterações da mesma possam se beneficiar do tratamento antiviral.

Os pacientes que usavam tenofovir tiveram prevalência maior de proteinúria e tendência à filtração glomerular menor que $60 \mathrm{ml} /$ $\mathrm{min} / 1.73 \mathrm{~m}^{2}$ (OR 2,1 p = 0,084), mas ambos sem significância estatística. É possível que o menor número de pacientes em uso de tenofovir em nosso estudo não tenha conferido ao mesmo poder de detectar este efeito. No entanto, o mais longo estudo de seguimento de uma coorte européia, recentemente, publicado ${ }^{22}$, também, não demonstrou diferença na evolução da filtração glomerular entre pacientes que usavam tenofovir e aqueles que não o usavam, ao contrário do observado em outros estudos ${ }^{23,24}$. Os pacientes que usavam lopinavir/r também mostravam maior frequência de filtração glomerular estimada abaixo de $60 \mathrm{ml} / \mathrm{min} / 1.73 \mathrm{~m}^{2}$ (OR 3,3: $\mathrm{p}=0,004)$, resultado que não permaneceu na análise de regressão. Recentemente, foi relatado em seguimento de 6.843 pacientes infectados pelo HIV-1, com pelo menos três estimativas consecutivas de filtração glomerular, que a exposição cumulativa ao tenofovir, indinavir, mas também ao atazanavir e ao lopinavir/r era associada a risco aumentado de doença renal crônica ${ }^{25}$. No caso específico do tenofovir, além de raros casos de síndrome de Fanconi, é possível que mesmo um risco de nefrotoxicidade não significativo, da ordem de $0,3 \%$ a $2 \%^{26}$, passe a se tornar mais observado já que é droga de primeira escolha em vários países da Europa e nos Estados Unidos da América. No Brasil, como o consenso do Ministério da Saúde o determina como medicação alternativa à zidovudina ${ }^{3}$, é possível que não tenha apresentado impacto em número de pacientes para ter significância na estatística de nosso estudo.
O acesso mais expandido aos antirretrovirais, se por um lado reduziu a mortalidade por doenças oportunistas, em razão do melhor controle viral e melhora dos níveis de linfócitos TCD4, por outro lado tem proporcionado aumento da expectativa de vida, com consequente aumento da mortalidade por causas não diretamente ligadas ao HIV-AIDS, como doenças renais ${ }^{27}$. Enfatizamos, assim, a importância do monitoramento da função renal em pacientes infectados pelo HIV-1, com atenção aos tradicionais fatores de risco para perda de função renal.

A população de nosso estudo ainda era relativamente jovem, com idade média de 42,76 (DP 10,29) anos, e tempo também não tão elevado de infecção pelo HIV-1, de 7,13 (DP 4,33) anos. Obviamente, não deve representar uma população com incidência elevada de doenças renais, o que destaca a importância de nossos achados. O envelhecimento da população infectada pelo HIV-1, o aumento da incidência em pessoas acima de 50 anos, e o caráter assintomático da doença renal, só fazem reforçar a importância do rastreamento cuidadoso da mesma como rotina, nos serviços de referência em nosso país.

\section{CONFLITO DE INTERESSE}

Os autores declaram não haver nenhum tipo de conflito de interesse no desenvolvimento do estudo.

\section{REFERÊNCIAS}

1. Pacheco AG, Saraceni V, Tuboi SH, Moulton LH, Chaisson RE, Cavalcante SC et al. Validation of a hierarchical deterministic Record-linkage algorithm using data from 2 different cohorts of human immunodeficency virus-infected persons and mortality databases in Brazil. Am J Epidemiol 2008; 168:1326-1332.

2. Ministério da Saúde. Secretaria de Vigilância Sanitária. Departamento de DST, AIDS e Hepatites Virais. Boletim Epidemiológico AIDS DST 2009. [Acesso 23 março 2010]. Disponível em: http://www.aids.gov.br/ data/documents/storedDocuments/\%7BB8EF5DAF-23AE-4891-AD361903553A3174\%7D/\%7B5E12D537-5F08-484B-9960-F54FE345DC31\%7D/ Boletim2009_preliminar.pdf/.

3. Ministério da Saúde. Secretaria de Vigilância Sanitária. Departamento de DST, AIDS e Hepatites Virais. Recomendações para terapia antirretroviral em adultos infectados pelo HIV-1 2008. [Acesso 23 março 2010]. Disponível em http// www.aids.gov.br/consensos.

4. Gupta SK, Eustace JA, Winston JA, Boydstun II, Ahuja TS, Rodriguez RA, et al. Guidelines for the management of chronic kidney disease in HIV-infected patients: Recommendations of the HIV medicine association of the infectious diseases society of America. Clin Infect Dis 2005; 40:1559-1585.

5. Gardenswartz MH, Lerner CW, Seligson GR, Zabetakis PM, Rotterdam H, Tapper ML, et al. Renal disease in patients with AIDS: A clinicopathologic study. Clin Nephrol 1984; 21:197-204.

6. Rao TK, Filippone EJ, Nicastri AD, Landesman SH, Frank E, Chen CK, et al. Associated focal and segmental glomerulosclerosis in the acquired immunodeficiency syndrome. N Engl J Med 1984; 310:669-673.

7. Atta MG. Diagnosis and natural history of HIV-associated nephropathy. Adv Chronic Kidney Dis 2010; 17:52-58

8. Ross MJ, Klotman PE. Recent progress in HIV associated nephropathy J Am Soc Nephrol 2002; 13:2997-3004.

9. Wyatt CM, Klotman PE. HIV-associated nephropathy in the era of antiretroviral therapy. Am J Med 2007; 120:488-492.

10. Fine DM, Atta MG. Kidney Disease in the HIV-infected patient. AIDS Patient Care and STDs 2007; 21:813-824.

11. Winston JA. HIV and CKD epidemiology. Adv Chronic Kidney Dis 2010; $17: 19-25$. 
12. Post FA, Holt SG. Recent developments in HIV and the kidney. Curr Opin Infect Dis 2009; 22:43-48.

13. Brown TT, Cole SR, LiX. Antiretroviral therapy and the prevalence and incidence of diabetes mellitus in the multicenter AIDS cohort study. Arch Intern Med 2005; 165:1179-1184.

14. Fine DM. Top HIV Med 2007; 14:164-169.

15. Winston JA. Assessing kidney function in HIV-infection. AIDS Read 2007; $17: 257-264$.

16. Gardner LI, Holmberg SD, Williamson JM. Development of proteinuria or elevated serum creatinine and mortality in HIV-infected women. J Acquir Immune Defic Syndr 2003; 32: 203-209.

17. Mochel A, Rosa TT, Veiga JPR. Aspectos da função renal em portadores do vírus da imunodeficiência humana. Rev Soc Bras Med Trop 1998; 31:179-186.

18. Ravasi G, Lauriola M, Tinelli C, Brandolini M, Uglietti A, Maserati R. Comparison of glomerular filtration rate estimates vs 24 -h creatinine clearance in HIV-positive patients. HIV Medicine 2009; 10:219-228.

19. Estrella MM, Fine DM. Screening for chronic kidney disease in HIV-infected patients. Adv Chronic Kidney Dis 2010; 17:26-35.

20. Hamzah L, Post FA. HIV and kidney disease. Medicine 2009; 37:365-367.

21. Fernando SK, Finkelstein FO, Moore BA, Weissman S. Prevalence of chronic kidney disease in a urban HIV infected population. Am J Med Sci 2008; 335:8994.

22. Leport C, Bouteloup V, Rossert J, Garré M, Iordache L, Dellamonica P, et al. Long-term evolution and determinants of renal function in HIV-infected patients who began receiving combination antiretroviral therapy in 19997-1999, ANRS CO8 APROCO-COPILOTE. Clin Infect Dis 2009; 49:1950-1954.

23. Fux CA, Simcock M, Wolbers M, Bucher HC, Hirschel B, Opravil M, et al. Tenofovir use is associated with a reduction in calculated glomerular filtration rates in the Swiss HIV Cohort Study. Antivir Ther 2007; 12:1165-1173.

24. Tan LKK, Gilleece Y, Mandalia S, Murungi A, Grover D, Fisher M, et al. Reduced glomerular filtration rate but sustained virological response in HIV/hepatitis B co-infected individuals on long term tenofovir. J Vir Hepatitis 2009; 16970:471478.

25. Kirk O, Mocroft A, Reiss P, De Wit S, Sedlacek D, Beniowski M, at al. Chronic Kidney Disease and Exposure to ART in a Large Cohort with Long-term Followup: The EuroSIDA Study. [Acesso em 09 abril 2010]. Disponível em: http:// www.retroconference.org/2010/Abstracts/39775.htm

26. Gupta SK. Tenofovir-associated Fanconi Syndrome: review of the FDA adverse event reporting system. Aids Patient Care STDs 2008; 22:99-103.

27. Fine DM, Perazella MA, Lucas GM, Atta MG. Renal Disease in patients with HIV infection. Epidemiology, pathogenesis and management. Drugs 2008; 68:963-980. 